\title{
INVARIANCE PRINCIPLES FOR THE LAW OF THE ITERATED LOGARITHM FOR MARTINGALES AND PROCESSES WITH STATIONARY INCREMENTS
}

\author{
By C. C. Heyde and D. J. Scott ${ }^{1}$ \\ Australian National University
}

\begin{abstract}
The main result in this paper is an invariance principle for the law of the iterated logarithm for square integrable martingales subject to fairly mild regularity conditions on the increments. When specialized to the case of identically distributed increments the result contains that of Stout [16] as well as the invariance principle for independent random variables of Strassen [17]. The martingale result is also used to obtain an invariance principle for the iterated logarithm law for a wide class of stationary ergodic sequences and a corollary is given which extends recent results of Oodaira and Yoshihara [10] on $\phi$-mixing processes.
\end{abstract}

1. Introduction. This paper is basically divided into two parts. In the first part a general invariance principle for the law of the iterated logarithm for martingales is obtained. This result (Theorem 1) specializes, in the case of identically distributed increments, to a result (Corollary 2) which contains the invariance principle of Strassen [17] for sums of independent and identically distributed random variables. Corollary 2 also contains Stout's [16] result giving a classical Hartman-Wintner form of iterated logarithm law for stationary ergodic martingales and its minor generalization by Heyde [6]. Our approach to Theorem 1 follows essentially the pattern of that of Theorem 3 of [17] in that it is based on the use of a form of the Skorokhod representation theorem.

In the second part of the paper we make use of Corollary 2 to establish an invariance principle for the law of the iterated logarithm for a certain class of (strictly) stationary processes (Theorem 2). The basic idea is a representation for the increments of the stationary process in terms of the increments of a stationary martingale plus other terms whose sum is negligible under suitable norming. This idea is due to Gordin [4], who has used it to prove a central limit theorem for stationary ergodic sequences and it has been further developed, in that context, by Scott [14]. As a corollary to Theorem 2 we obtain Corollary 3 which extends results of Oodaira and Yoshihara [10], who have given an invariance principle for the law of the iterated logarithm for stationary $\phi$-mixing sequences.

Previous work on iterated logarithm results for stationary processes has proceeded via classical methods. Our approach via the Skorokhod representation is

Received March 27, 1972; revised October 6, 1972.

${ }_{1}$ Now at La Trobe University.

AMS 1970 subject classifications. Primary: 60B10, 60F15, Secondary: 60G10, 60G45.

Key words and phrases. Invariance principles, iterated logarithm law, martingales, stationary ergodic processes, $\phi$-mixing. 
more direct, gives ready access to the invariance principle, and avoids the necessity of obtaining detailed estimates of probabilities via, for example, rates of convergence to normality. In principle the result of Theorem 2 could be obtained by classical methods by paralleling the work of Chover [2] but a prerequisite would be estimates of rates of convergence to normality along the lines of those of Heyde [5] for sums of independent random variables.

2. Martingale results. Let $\left\{S_{n}, \mathscr{I}_{n} ; n \geqq 0\right\}$ be a martingale on the probability space $(\Omega, \mathscr{A}, p)$ where $\mathscr{F}_{0}=\{\phi, \Omega\}$ and $\mathscr{J}_{n}=\sigma$-field generated by $S_{1}, S_{2}, \ldots$, $S_{n}$ for $n>0$. Let $S_{0}=X_{0}=0$ almost surely (a.s.) and $S_{n}=\sum_{k=1}^{n} X_{k}$ for $n \geqq 1$. Further, let $s_{n}{ }^{2}=E S_{n}{ }^{2}<\infty$.

We consider the metric space $(C, \rho)$ of all real-valued continuous functions on $[0,1]$ with

$$
\rho(x, y)=\sup _{0 \leq t \leq 1}|x(t)-y(t)| \quad \text { for } x, y \in C .
$$

Let $K$. be the set of absolutely continuous $x \in C$ such that $x(0)=0$ and

$$
\int_{0}^{1}[\dot{x}(t)]^{2} d t \leqq 1
$$

where $\dot{x}$ denotes the derivative of $x$ determined almost everywhere with respect to Lebesgue measure. Define a real function $g(\cdot)$ on $[0, \infty)$ by

$$
g(t)=\sup \left\{n: s_{n}^{2} \leqq t\right\} .
$$

Let $\xi$ be a standard Wiener process (Brownian motion) on $[0, \infty)$ and define a sequence of real random functions $\xi_{n}(\cdot)$ on $[0,1]$, for $n>g(e)$ by

$$
\xi_{n}(t)=\left[\phi\left(s_{n}{ }^{2}\right)\right]^{-1} \xi\left(s_{n}{ }^{2} t\right) \quad t \in[0,1]
$$

where $\phi(\cdot)$ is a real function on $[e, \infty)$ given by

$$
\phi(t)=(2 t \log \log t)^{\frac{1}{2}} \quad t \in[e, \infty) .
$$

We also define a sequence of real random functions $\eta_{n}(\cdot)$ on $[0,1]$, for $n>g(e)$, by

$$
\begin{aligned}
& \eta_{n}(t)=\left[\phi\left(s_{n}{ }^{2}\right)\right]^{-1}\left[S_{k}+\left(s_{n}{ }^{2} t-s_{k}{ }^{2}\right)\left(s_{k+1}^{2}-s_{k}{ }^{2}\right)^{-1} X_{k+1}\right] \\
& s_{k}^{2} \leqq s_{n}^{2} t \leqq s_{k+1}^{2}, \quad k=0,1, \cdots, n-1 .
\end{aligned}
$$

THEOREM 1. If $s_{n}{ }^{2} \rightarrow \infty$ and

$$
\begin{array}{rr}
\sum_{n=1}^{\infty} s_{n}{ }^{-4} E\left\{X_{n}^{4} I\left(\left|X_{n}\right|<\delta s_{n}\right)\right\}<\infty & \text { for some } \delta>0, \\
\sum_{n=1}^{\infty} s_{n}{ }^{-1} E\left\{\left|X_{n}\right| I\left(\left|X_{n}\right| \geqq \varepsilon s_{n}\right)\right\}<\infty & \text { for all } \varepsilon>0, \\
s_{n}{ }^{-2} \sum_{k=1}^{n} X_{k}^{2} \rightarrow_{\text {a.s. }} 1 & \text { as } n \rightarrow \infty
\end{array}
$$

hold, $I(\cdot)$ denoting the indicator function, then $\left\{\eta_{n} ; n>g(e)\right\}$ is relatively compact

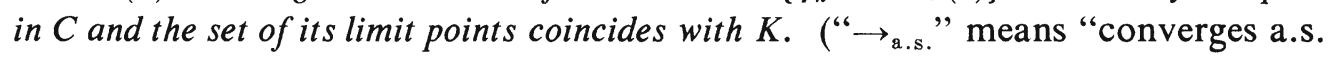
to.")

Corollary 1. If $s_{n}{ }^{2} \rightarrow \infty$ as $n \rightarrow \infty$,

$$
\sum_{n=1}^{\infty} s_{n}^{-4} E X_{n}^{4}<\infty
$$


and (3) holds then $\left\{\eta_{n} ; n>g(e)\right\}$ is relatively compact in $C$ and the set of its limit points coincides with $K$.

COROLLARY 2. If the $X_{n}$ are identically distributed and satisfy (3), then $\left\{\eta_{n} ; n>\right.$ $\left.e\left[E\left(X_{1}^{2}\right)\right]^{-1}\right\}$ is relatively compact in $C$ and the set of its limit points coincides with $K$.

The condition (3) is, unfortunately, often difficult to check. It holds, for example, if $\left\{X_{n}\right\}$ is a stationary ergodic sequence.

The result of Theorem 1 appears to be new even in the case of independent random variables. Other iterated logarithm type results for martingales have been obtained by Stout [15] and Strassen [18] but they involve random norming and do not seem to be directly comparable with the present results.

3. Martingale proofs. The first step is to define a truncated martingale sequence $\left\{S_{n}{ }^{*}, \mathscr{J}_{n}{ }^{*} ; n \geqq 0\right\}$. For $\delta>0$, let $X_{0}{ }^{*}=0$ a.s., $\mathscr{F}_{0}{ }^{*}=\{\phi, \Omega\}$ and for $k>0$,

$$
X_{k}^{*}=X_{k} I\left(\left|X_{k}\right|<\delta s_{k}\right)-E\left\{X_{k} I\left(\left|X_{k}\right|<\delta s_{k}\right) \mid \mathscr{J}_{k-1}^{*}\right\}
$$

where $\mathscr{F}_{k}{ }^{*}=\sigma$-field generated by $X_{1}{ }^{*}, \cdots, X_{k}{ }^{*}$. Then we let

$$
S_{n}^{*}=\sum_{k=0}^{n} X_{k}^{*} \quad \text { for } n \geqq 0 \text {, }
$$

and $s_{n}{ }^{2}=E S_{n}{ }^{2} \leqq s_{n}{ }^{2}<\infty$. For $n>g(e)$ we define a sequence of real random functions $\eta_{n}^{*}(\cdot)$ on $[0,1]$ by

$$
\begin{aligned}
\eta_{n}{ }^{*}(t)=\left[\phi\left(s_{n}{ }^{2}\right)\right]^{-1}\left[S_{k}{ }^{*}+\left(s_{n}{ }^{2} t-s_{k}{ }^{2}\right)\left(s_{k+1}^{2}-{s_{k}}^{2}\right)^{-1} X_{k+1}^{*}\right] \\
s_{k}{ }^{2} \leqq t s_{n}{ }^{2} \leqq s_{k+1}^{2}, k=0,1, \cdots, n-1 .
\end{aligned}
$$

We first observe that

$$
\sup _{0 \leqq t \leqq 1}\left|\eta_{n}{ }^{*}(t)-\eta_{n}(t)\right| \rightarrow_{\mathrm{a} . \mathrm{s} .} 0
$$

as $n \rightarrow \infty$

under the conditions of Theorem 1. This will of course be true if

$$
s_{n}^{-1} \sum_{k=1}^{n}\left|X_{k}\right| I\left(\left|X_{k}\right| \geqq \delta s_{k}\right) \stackrel{\text { ’ }}{\mathrm{a} . \mathrm{s} .} 0 \quad \text { as } n \rightarrow \infty
$$

and

$$
s_{n}^{-1} \sum_{k=1}^{n}\left|E\left\{X_{k} I\left(\left|X_{k}\right|<\delta s_{k}\right) \mid \mathscr{J}_{k-1}^{*}\right\}\right| \rightarrow_{\text {a.s. }} 0 \quad \text { as } n \rightarrow \infty,
$$

and these results follow straightforwardly from (2) via the Kronecker lemma and the monotone convergence theorem.

Now we introduce the Skorokhod representation. From Theorem 4.3 of Strassen [18] we may conclude that there exists a sequence $T_{0}=0, T_{1}, T_{2}, \ldots$ of nonnegative random variables such that

$$
\left(\bar{S}_{1}, \bar{S}_{2}, \cdots, \bar{S}_{n}\right) \equiv\left(\xi\left(T_{1}\right), \xi\left(T_{1}+T_{2}\right), \cdots, \xi\left(\sum_{k=1}^{n} T_{k}\right)\right)
$$

is distributed as

$$
\left(S_{1}^{*}, S_{2}^{*}, \cdots, S_{n}^{*}\right)
$$

for each $n \geqq 1$. Further, if $\bar{S}_{0}=0$ a.s., $\overline{\mathscr{F}}_{0}=\{\phi, \Omega\}, \overline{\mathscr{I}}_{n}$ for $n \geqq 1$ is the $\sigma$ field generated by $\bar{S}_{1}, \cdots, \bar{S}_{n}, \mathscr{G}_{0}=\mathscr{I}_{0}, \mathscr{G}_{n}$ is the $\sigma$-field generated by $\xi(t)$ for 
$0 \leqq t \leqq \sum_{k=1}^{n} T_{k}$ for $n \geqq 1$ and $\bar{S}_{n}=\sum_{k=0}^{n} \bar{X}_{k}$ for $n \geqq 0$, then

$$
E\left\{T_{n} \mid \mathscr{G}_{n-1}\right\}=E\left\{\bar{X}_{n}{ }^{2} \mid \overline{\mathscr{J}}_{n-1}\right\} \text { a.s. } \quad \text { for } n \geqq 1 \text {. }
$$

Also, for $r>1$,

$$
E\left\{T_{n}{ }^{r} \mid \mathscr{G}_{n-1}\right\} \leqq L_{r} E\left\{\bar{X}_{n}{ }^{2 r} \mid \overline{\mathscr{J}}_{n-1}\right\} \quad \text { a.s. } \quad \text { for } n \geqq 1,
$$

where $L_{r}$ is a constant depending only on $r$.

Define $\bar{\eta}_{n}(\cdot)$ on $[0,1]$, for $n>g(e)$, by

$$
\begin{aligned}
\tilde{\eta}_{n}(t)=\left[\phi\left(s_{n}{ }^{2}\right)\right]^{-1}\left[\bar{S}_{k}+\left(s_{n}{ }^{2} t-s_{k}{ }^{2}\right)\left(s_{k+1}^{2}-s_{k}{ }^{2}\right)^{-1} \bar{X}_{k+1}\right] \\
s_{k}{ }^{2} \leqq s_{n}{ }^{2} t \leqq s_{k+1}^{2}, k=0,1, \ldots, n-1,
\end{aligned}
$$

and $\eta(\cdot)$ on $[0, \infty)$ by

Then

$$
\eta(t)=\bar{S}_{n}+\bar{X}_{n+1}\left(t-s_{n}^{2}\right)\left(s_{n+1}^{2}-s_{n}^{2}\right)^{-1} \quad s_{n}^{2} \leqq t \leqq s_{n+1}^{2} .
$$

for any $n>g(e)$.

$$
\eta(t)=\phi\left(s_{n}{ }^{2}\right) \bar{\eta}_{n}\left(s_{n}{ }^{-2} t\right) \quad \text { for } t \in\left[0, s_{n}{ }^{2}\right]
$$

Our proof follows the work of Strassen in [17]. In fact, in view of (5) and (8) above and the results of [17], Theorem 1 will follow provided we can show that $s_{n+1}^{2} / s_{n}{ }^{2} \rightarrow 1$ and $\rho\left(\tilde{\eta}_{n}, \xi_{n}\right) \rightarrow_{\text {a.s. }} 0$ and this last condition is implied by

$$
P\left(\lim _{t \rightarrow \infty}[\phi(t)]^{-1} \sup _{\tau \leqq t}|\eta(\tau)-\xi(\tau)|=0\right)=1 .
$$

In order to prove that $s_{n+1}^{2} / s_{n}{ }^{2} \rightarrow 1$, we first note that (2) gives, via an application of the Kronecker Lemma,

$$
s_{n}^{-1} \sup _{k \leqq n}\left|X_{k}\right| I\left(\left|X_{k}\right| \geqq \varepsilon s_{k}\right) \rightarrow_{\text {a.s. }} 0, \quad \text { for any } \varepsilon>0,
$$

so that upon squaring we have $a$ fortiori

Thus

$$
s_{n}{ }^{-2} \sup _{k \leqq n} X_{k}^{2} I\left(\left|X_{k}\right| \geqq \varepsilon s_{n}\right) \rightarrow_{\text {a.s. }} 0 .
$$

$$
s_{n}^{-2} \sup _{k \leqq n} X_{k}^{2} \leqq \varepsilon^{2}+s_{n}^{-2} \sup _{k \leqq n} X_{k}^{2} I\left(\left|X_{k}\right| \geqq \varepsilon S_{n}\right),
$$

and since $\varepsilon$ may be chosen arbitrarily small,

$$
s_{n}{ }^{-2} \sup _{k \leqq n} X_{k}{ }^{2} \rightarrow_{\text {a.s. }} 0 .
$$

From (3) and Theorm 1 of Pratt [12] we then deduce that

$$
\lim _{n \rightarrow \infty} s_{n}^{-2} \sup _{k \leqq n} E X_{k}^{2} \leqq \lim _{n \rightarrow \infty} E s_{n}^{-2} \sup _{k \leqq n} X_{k}^{2}=0,
$$

and $s_{n+1}^{2} / s_{n}{ }^{2} \rightarrow 1$ follows.

We next proceed to the proof of (11) for which we need to know more about the behavior of the sequence $T_{1}, T_{2}, \ldots$.

Lemma 1. Under the conditions of Theorem 1,

$$
s_{n}^{-2} \sum_{k=1}^{n} E\left\{T_{k} \mid \mathscr{G}_{k-1}\right\} \rightarrow_{\text {a.s. }} 1 \quad \text { as } n \rightarrow \infty .
$$

Proof. Using (81 and (9), we need only show

$$
s_{n}{ }^{-2} \sum_{k=1}^{n} E\left\{X_{k}{ }^{2} \mid \mathscr{I}_{k-1}^{*}\right\} \rightarrow_{\text {a.s. }} 1 \quad \text { as } n \rightarrow \infty .
$$


Now

$$
\begin{aligned}
& s_{n}{ }^{-2} \sum_{k=1}^{n} E\left\{X_{k}{ }^{2} \mid \mathscr{F}_{k-1}^{*}\right\} \\
& \quad=s_{n}{ }^{-2} \sum_{k=1}^{n}\left[E\left\{X_{k}^{2} I\left(\left|X_{k}\right|<\delta s_{k}\right) \mid \mathscr{I}_{k-1}^{*}\right\}-\left(E\left\{X_{k} I\left(\left|X_{k}\right|<\delta s_{k}\right) \mid \mathscr{J}_{k-1}^{*}\right\}\right)^{2}\right]
\end{aligned}
$$

and

$$
\begin{aligned}
s_{n}{ }^{-2} \sum_{k=1}^{n} & \left(E\left\{X_{k} I\left(\left|X_{k}\right|<\delta s_{k}\right) \mid \mathscr{F}_{k-1}^{*}\right\}\right)^{2} \\
& \leqq s_{n}{ }^{-1} \delta \sum_{k=1}^{n} E\left\{\left|X_{k}\right| I\left(\left|X_{k}\right| \geqq \delta s_{n}\right) \mid \mathscr{J}_{k-1}^{*}\right\} \rightarrow_{\mathrm{a} . \mathrm{s} .} 0 \quad \text { as } n \rightarrow \infty
\end{aligned}
$$

Also, using Proposition IV. 6.1, page 147 of Neveu [8],

$$
\begin{array}{r}
s_{n}^{-2} \sum_{k=1}^{n}\left(X_{k}^{2} I\left(\left|X_{k}\right|<\delta s_{k}\right)-E\left\{X_{k}^{2} I\left(\left|X_{k}\right|<\delta s_{k}\right) \mid \mathscr{F}_{k-1}^{*}\right\}\right) \rightarrow_{\text {a.s. }} 0 \\
\text { as } n \rightarrow \infty
\end{array}
$$

since

$$
\begin{aligned}
\sum_{n=1}^{\infty} s_{n}{ }^{-4} E\left[X_{n}{ }^{2} I\left(\left|X_{n}\right|<\delta s_{n}\right)\right. & \left.-E\left\{X_{n}{ }^{2} I\left(\left|X_{n}\right|<\delta s_{n}\right) \mid \mathscr{I}_{n-1}^{*}\right\}\right]^{2} \\
& \leqq \sum_{n=1}^{\infty} s_{n}{ }^{-4} E\left[X_{n}{ }^{4} I\left(\left|X_{n}\right|<\delta s_{n}\right)\right]<\infty
\end{aligned}
$$

using (1). Consequently, from (15), (16) and (17), it suffices to show that

$$
s_{n}^{-2} \sum_{k=1}^{n} X_{k}^{2} I\left(\left|X_{k}\right|<\delta s_{k}\right) \rightarrow_{\text {a.s. }} 1
$$

as $n \rightarrow \infty$

or equivalently, in view of (3),

$$
s_{n}^{-2} \sum_{k=1}^{n} X_{k}^{2} I\left(\left|X_{k}\right| \geqq \delta s_{k}\right) \rightarrow_{\text {a.s. }} 0 \text {. }
$$

However,

$$
s_{n}{ }^{-2} \sum_{k=1}^{n} X_{k}{ }^{2} I\left(\left|X_{k}\right| \geqq \delta s_{k}\right) \leqq\left(s_{n}{ }^{-1} \sup _{k \leqq n}\left|X_{k}\right|\right) s_{n}{ }^{-1} \sum_{k=1}^{n}\left|X_{k}\right| I\left(\left|X_{k}\right| \geqq \delta s_{k}\right)
$$

and (18) follows from (2) and (12).

LEMMA 2. Under the conditions of Theorem 1,

$$
s_{n}{ }^{-2} \sum_{k=1}^{n}\left(T_{k}-E\left\{T_{k} \mid \mathscr{G}_{k-1}\right\}\right) \rightarrow_{\text {a.s. }} 0 \quad \text { as } n \rightarrow \infty .
$$

Proof. Again using Proposition IV. 6.1, page 147 of [8], the result will follow if

$$
\sum_{n=1}^{\infty} s_{n}{ }^{-4} E\left(T_{n}-E\left\{T_{n} \mid \mathscr{G}_{n-1}\right\}\right)^{2}<\infty .
$$

Now

$$
\begin{array}{rl}
\sum_{n=1}^{\infty} s_{n}{ }^{-4} & E\left(T_{n}-E\left\{T_{n} \mid \mathscr{G}_{n-1}\right\}\right)^{2} \\
\leqq & \sum_{n=1}^{\infty} s_{n}{ }^{-4} E T_{n}{ }^{2} \\
\leqq & L_{2} \sum_{n=1}^{\infty} s_{n}{ }^{-4} E X_{n}{ }^{4} \\
= & L_{2} \sum_{n=1}^{\infty} s_{n}{ }^{-4} E\left(X_{n} I\left(\left|X_{n}\right|<\delta s_{n}\right)-E\left\{X_{n} I\left(\left|X_{n}\right|<\delta s_{n}\right) \mid \mathscr{J}_{n-1}^{*}\right\}\right)^{4} \\
\leqq & L_{2} \sum_{n=1}^{\infty} s_{n}{ }^{-4}\left[E\left(X_{n}{ }^{4} I\left(\left|X_{n}\right|<\delta s_{n}\right)\right)\right. \\
& \left.\quad+15 \delta^{3} s_{n}{ }^{3} E\left|E\left\{X_{n} I\left(\left|X_{n}\right|<\delta s_{n}\right) \mid \mathscr{J}_{n-1}^{*}\right\}\right|\right] \\
\leqq & L_{2} \sum_{n=1}^{\infty} s_{n}{ }^{-4}\left[E\left(X_{n}{ }^{4} I\left(\left|X_{n}\right|<\delta s_{n}\right)\right)+15 \delta^{3} s_{n}{ }^{3} E\left(\left|X_{n}\right| I\left(\left|X_{n}\right| \geqq \delta s_{n}\right)\right)\right] \\
< & \infty
\end{array}
$$

by (1) and (2). 
We are now in a position to complete the proof of Theorem 1. Since $E X_{k}^{2}<\infty$ for any $k$,

$$
g(t) \rightarrow \infty \quad \text { as } \quad t \rightarrow \infty .
$$

Then also

$$
\begin{aligned}
\left|t s_{g(t)}^{-2}-1\right| & \leqq\left|s_{g(t)}^{-2} E X_{g(t)+1}^{2}\right| \\
& =\left|s_{g(t)+1}^{-2} E X_{g(t)+1}^{2}\right|\left|s_{g(t)+1}^{2} s_{g(t)}^{-2}\right| \\
& \rightarrow 0
\end{aligned}
$$$$
\text { as } t \rightarrow \infty
$$

by (13) and (19). Similarly,

$$
t s_{g(t)+1}^{-2} \rightarrow 1
$$

as $t \rightarrow \infty$.

From (19), (20), (21) and Lemmas 1 and 2 we obtain

and

$$
t^{-1} \sum_{\substack{g(t) \\ k=1}} T_{k} \rightarrow_{\text {a.s. }} 1 \quad \text { as } t \rightarrow \infty
$$

Finally, we note that

$$
t^{-1} \sum_{k=1}^{g(t)+1} T_{k} \rightarrow_{\text {a.s. }} 1 \quad \text { as } t \rightarrow \infty .
$$

$$
|\eta(t)-\xi(t)| \leqq \max \left\{\left|\xi\left(\sum_{k=1}^{g(t)} T_{k}\right)-\xi(t)\right|,\left|\xi\left(\sum_{k=1}^{g(t)+1} T_{k}\right)-\xi(t)\right|\right\} .
$$

From this point, it is obvious that Strassen's proof on page 217 of [17] may be followed to obtain (11), which completes the proof of Theorem 1 .

Corollaries 1 and 2 follow easily from Theorem 1 using standard arguments of the kind of, for example, Neveu [8], pages 153, 154.

4. Stationary process results. We consider a probability space $(\Omega, \mathscr{A}, P)$ with an ergodic automorphism $T$. Let $L_{2}(P)$ be the Hilbert space of random variables with finite second moment, and write $\left(E X^{2}\right)^{\frac{1}{2}}$ as $\|X\|$. Define $U$ on $L_{2}(P)$ by $U X(\omega)=X(T \omega)$ for $X \in L_{2}(P), \omega \in \Omega$. Then $U$ is a unitary operator. If $\mathscr{L}_{0}$ is a $\sigma$-field such that $\mathscr{M}_{0} \subset \mathscr{A}$ and $\mathscr{M}_{0} \subset T^{-1}\left(\mathscr{M}_{0}\right)$, define $\mathscr{M}_{k}=T^{-k}\left(\mathscr{M}_{0}\right)$, $\mathscr{M}_{-\infty}=\bigcap_{k=-\infty}^{\infty} \mathscr{M}_{k}$ and $\mathscr{M}_{+\infty}=\sigma$-field generated by $\bigcup_{k=-\infty}^{\infty} \mathscr{M}_{k}$.

We consider a particular random variable $X_{0} \in L_{2}(P)$. Defining $X_{k}=U^{k} X_{0}$, we see that $\cdots, X_{-1}, X_{0}, X_{1}, \cdots$ is a doubly infinite stationary ergodic sequence. Put $X^{(0)}=0$ and $X^{(n)}=\sum_{k=0}^{n-1} X_{k}$ for $n \geqq 1$ and define a sequence of random functions $\theta_{n}(\cdot)$ on $[0,1]$ by

Also define

$$
\begin{array}{r}
\theta_{n}(t)=\left[\phi\left(\left\|X^{(n)}\right\|^{2}\right)\right]^{-1}\left(X^{(k)}+(n t-k) X_{k}\right) \\
k \leqq n t \leqq k+1, k=0,1, \ldots, n-1 .
\end{array}
$$

THEOREM 2. If

$$
g=\sup \left(n:\left\|X^{(n)}\right\|^{2} \leqq e\right\} .
$$

$$
\sum_{m=0}^{\infty}\left(\left\|E\left\{X_{0} \mid \mathscr{M}_{-m}\right\}\right\|+\left\|X_{0}-E\left\{X_{0} \mid \mathscr{M}_{m}\right\}\right\|\right)<\infty
$$

then

$$
\lim _{n \rightarrow \infty}\left\|X^{(n)}\right\| / n^{\frac{1}{2}}=\sigma
$$


exists for $0 \leqq \sigma<\infty$. If $\sigma>0$ then $g<\infty,\left\{\theta_{n} ; n>g\right\}$ is relatively compact and the set of its limit points coincides with $K$.

Proof. The key to the proof is a representation

$$
X_{0}=Y_{0}+U Z_{0}-Z_{0}
$$

where $Y_{0}, Z_{0}$ belong to $L_{2}(P)$ and, writing $Y_{k}=U^{k} Y_{0}, Y^{(0)}=0$ and $Y^{(n)}=$ $\sum_{k=1}^{n-1} Y_{k}$ for $k \geqq 1,\left\{Y^{(n)}, \mathscr{M}_{n-1} ; n \geqq 0\right\}$ is a stationary ergodic zero-mean martingale. This representation, which is due to Gordin, holds under the condition (22); see Scott [14] for full details.

Setting $\left\|Y_{0}\right\|=\sigma$ we have $\left\|Y^{(n)}\right\|=\sigma n^{\frac{1}{2}}$. Also, from Minkowski's inequality and (23),

$$
\left\|X^{(n)}\right\|-\sigma n^{\frac{1}{2}} \mid / n^{\frac{1}{2}} \leqq 2\left\|Z_{0}\right\| / n^{\frac{1}{2}} \rightarrow 0
$$

since $Z_{0} \in L_{2}(P)$ and thus

$$
\lim _{n \rightarrow \infty}\left\|X^{(n)}\right\| / n^{\frac{1}{2}}=\sigma .
$$

We henceforth assume $\sigma>0$. Then

$$
\lim _{n \rightarrow \infty}\left\|X^{(n)}\right\| / \sigma n^{\frac{1}{2}}=1
$$

and $g<\infty$. Also, from (24) we readily deduce that

$$
\phi\left(\left\|X^{(n)}\right\|^{2}\right) / \phi\left(n \sigma^{2}\right) \rightarrow 1 .
$$

Now define a sequence of random functions $\zeta_{n}(\cdot)$ on $[0,1]$ by

$$
\begin{aligned}
\zeta_{n}(t)=\left[\phi\left(n \sigma^{2}\right)\right]^{-1}\left[Y^{(k)}+(n t-k) Y_{k}\right] \\
k \leqq n t \leqq k+1, k=0,1, \cdots, n-1 .
\end{aligned}
$$

Then by Corollary 2 we know that $\left\{\zeta_{n} ; n>e / \sigma^{2}\right\}$ is relatively compact and the set of its limit points coincides with $K$. Thus, the proof will be complete if we can show that

$$
\sup _{0 \leqq t \leqq 1}\left|\theta_{n}(t)-\zeta_{n}(t)\right| \rightarrow_{\text {a.s. }} 0
$$

We first consider

Now

$$
\begin{aligned}
\sup _{0 \leqq t \leqq 1} \mid \zeta_{n}(t)-\phi\left(n \sigma^{2}\right)[\phi & \left.\left(|| X^{(n)}||^{2}\right)\right]^{-1} \zeta_{n}(t) \mid \\
& \leqq\left|1-\phi\left(n \sigma^{2}\right)\left[\phi\left(|| X^{(n)}||^{2}\right)\right]^{-1}\right| \sup _{0 \leqq t \leqq 1}\left|\zeta_{n}(t)\right|
\end{aligned}
$$

$$
\begin{aligned}
\lim \sup _{n \rightarrow \infty} \sup _{0 \leqq t \leqq 1}\left|\zeta_{n}(t)\right| & =\sup _{x \in K} \sup _{0 \leqq t \leqq 1}|x(t)| \text { a.s. } \\
& \leqq 1 \text { a.s. }
\end{aligned}
$$

From this and (25) and (27) we conclude that

$$
\sup _{0 \leqq t \leqq 1}\left|\zeta_{n}(t)-\phi\left(n \sigma^{2}\right)\left[\phi\left(\left\|X^{(n)}\right\|^{2}\right)\right]^{-1} \zeta_{n}(t)\right| \rightarrow_{\text {a.s. }} 0 .
$$

Now, writing $Z_{k}=U^{k} Z_{0}$, consider

$$
\begin{aligned}
\sup _{0 \leqq t \leqq 1} \mid \theta_{n}(t) & -\phi\left(n \sigma^{2}\right)\left[\phi\left(\| X^{(n)}||^{2}\right)\right]^{-1} \zeta_{n}(t) \mid \\
& =\sup _{1 \leqq k \leqq n}\left[\phi\left(\left\|X^{(n)}\right\|^{2}\right)\right]^{-1}\left|Z_{k}-Z_{0}\right| \\
& \leqq 2 \sup _{0 \leqq k \leqq n}\left[\phi\left(\left\|X^{(n)}\right\|^{2}\right)\right]^{-1}\left|Z_{k}\right|
\end{aligned}
$$


which goes almost surely to zero provided

$$
\sup _{0 \leqq k \leqq n}\left[\phi\left(n \sigma^{2}\right)\right]^{-1}\left|Z_{k}\right|=\left(n \sigma^{2} \log \log n \sigma^{2}\right)^{-\frac{1}{2}} \sup _{0 \leqq k \leqq n}\left|Z_{k}\right| \rightarrow_{\text {a.s. }} 0
$$

(by (25)) and thus if

$$
(n \log \log n)^{-\frac{1}{2}}\left|Z_{n}\right| \rightarrow_{\text {a.s. }} 0 .
$$

But, this last result follows from the Borel-Cantelli Lemma since $Z_{0} \in L_{2}(P)$. Thus

$$
\sup _{0 \leqq t \leqq 1}\left|\theta_{n}(t)-\phi\left(n \sigma^{2}\right)\left[\phi\left(\|\left. X^{(n)}\right|^{2}\right)\right]^{-1} \zeta_{n}(t)\right| \rightarrow_{\text {a.s. }} 0
$$

and taking this with (28), (26) follows and the proof is complete.

As an application of Theorem 2, we shall see how it extends some recent results of Oodaira and Yoshihara [9], [10]. The papers [9], [10] in turn improve results of Iosifescu [7] and Reznik [13] as well as the (strictly) stationary case of results of Philipp [11].

Let $\left\{X_{j},-\infty<j<\infty\right\}$ be a stationary process defined on a probability space $(\Omega, \mathscr{A}, P)$. Write $\mathscr{M}_{k}$ for the $\sigma$-field generated by $\cdots, X_{k-1}, X_{k} ; \mathscr{M}_{\infty}$ for the $\sigma$-field generated by $\cdots, X_{-1}, X_{0}, X_{1}, \cdots$ and $\mathscr{M}_{k}^{\infty}$ for the $\sigma$-field generated by $X_{k}, X_{k+1}, \cdots$ The sequence $\left\{X_{j}\right]$ is called $\phi$-mixing if for each $k(-\infty<k<\infty)$ and each $n(n \geqq 1)$,

$$
\sup _{B \in \mathscr{M}_{k+n}^{\infty}} \operatorname{ess} \sup \left|P\left(B \mid \mathscr{M}_{k}\right)-P(B)\right| \leqq \phi_{n} \downarrow 0 \quad \text { as } n \rightarrow \infty .
$$

Corollary 3. If $\left\{X_{n}\right\}$ is a stationary $\phi$-mixing process with $E X_{0}=0$, $E\left|X_{0}\right|^{2+\delta}<\infty$ some $\delta \geqq 0$ and $\sum_{n=1}^{\infty}\left[\phi_{n}\right]^{(1+\delta) /(2+\delta)}<\infty$, then

$$
\lim _{n \rightarrow \infty}\left\|X^{(n)}\right\|^{2} / n=E X_{0}^{2}+2 \sum_{i=1}^{\infty} E X_{0} X_{i}=\sigma^{2} \geqq 0 \text {. }
$$

If $\sigma>0$ then $g<\infty$ and $\left\{\theta_{n} ; n>g\right\}$ is relatively compact and the set of its limit points coincides with $K$.

Proof. First note that $\left\{X_{n}\right\}$ is stationary and $\phi$-mixing and hence ergodic. Further, there exists an ergodic transformation $T$ on $\mathscr{M}_{\infty}$ such that $X_{k}(\omega)=$ $X_{0}\left(T^{k} \omega\right), \omega \in \Omega$ (e.g. Doob [3], Chapter $X$ ) and, since $\mathscr{M}_{0}$ is the $\sigma$-field generated by $\cdots, X_{-1}, X_{0}$ our present situation fits within the framework of Theorem 2 .

Next we need to check (22). This is a simple matter using Lemma 1, page 170 of Billingsley [1]. We have

and

$$
\sum_{m=0}^{\infty}\left(\left\|E\left\{X_{0} \mid \mathscr{M}_{-m}\right\}\right\|+\left\|X_{0}-E\left\{X_{0} \mid \mathscr{M}_{m}\right\}\right\|\right)=\sum_{m=0}^{\infty}\left\|E\left\{X_{0} \mid \mathscr{M}_{-m}\right\}\right\|
$$

$$
\begin{aligned}
E\left[E\left\{X_{0} \mid \mathscr{M}_{-m}\right\}\right]^{2} & =E\left[X_{0} E\left\{X_{0} \mid \mathscr{M}_{-m}\right\}\right] \\
& \leqq 2 \phi_{m}{ }^{(1+\delta) /(2+\delta)}\left[E\left|E\left\{X_{0} \mid \mathscr{M}_{-m}\right\}\right|^{(2+\delta) /(1+\delta)}\right]^{(1+\delta) /(2+\delta)}\left[E\left|X_{0}\right|^{2+\delta}\right]^{1 /(2+\delta)} \\
& \leqq 2 \phi_{m}{ }^{(1+\delta) /(2+\delta)}\left[E\left[E\left\{X_{0} \mid \mathscr{M}_{-m}\right\}\right]^{2}\right]^{\frac{1}{2}}\left[E\left|X_{0}\right|^{2+\delta}\right]^{1 /(2+\delta)}
\end{aligned}
$$

so that

$$
\left\|E\left\{X_{0} \mid \mathscr{M}_{-m}\right\}\right\| \leqq 2 \phi_{m}^{(1+\delta) /(2+\delta)}\left[E\left|X_{0}\right|^{2+\delta}\right]^{1 /(2+\delta)}
$$

and (22) holds under the conditions of the corollary. 
Finally, from Lemma 3, page 172 of [1],

$$
\lim _{n \rightarrow \infty}\left\|X^{(n)}\right\|^{2} / n=E X_{0}^{2}+2 \sum_{i=1}^{\infty} E\left(X_{0} X_{i}\right)
$$

and the corollary follows from Theorem 2.

In Theorem 1 of Oodaira and Yoshihara [10], an invariance principle for the law of the iterated logarithm is given under the conditions of Corollary 3 plus the requirements that $\int_{|x| \geqq N} x^{2} d P\left(X_{0} \leqq x\right)=O\left((\log N)^{-5}\right)$ as $N \rightarrow \infty$ if $\delta=0$ or $\phi_{n}=O\left(n^{-1-\varepsilon}\right)$ for some $\varepsilon>(1+\delta)^{-1}$ if $\delta>0$.

\section{REFERENCES}

[1] Billingsley, P. (1968). Convergence of Probability Measures. Wiley, New York.

[2] Chover, J. (1967). On Strassen's version of the log log law. Z. Wahrscheinlichkeitstheorie und Verw. Gebiete 8 83-90.

[3] Dоoв, J. L. (1953). Stochastic Processes. Wiley, New York.

[4] Gordin, M. I. (1969). The central limit theorem for stationary processes. Soviet Math. Dokl. 10 1174-1176.

[5] Heyde, C. C. (1969). Some properties of metrics in a study on convergence to normality. Z. Wahrscheinlichkeitstheorie und Verw. Gebiete 11 181-192.

[6] Heyde, C. C. (1971). An iterated logarithm result for martingales and its application in estimation theory for autoregressive processes. To appear in J. Appl. Probability 9.

[7] Iosifescu, M. (1968). The law of the iterated logarithm for a class of dependent random variables. Theor. Probability Appl. 13 304-313.

[8] Neveu, J. (1965). Mathematical Foundations of the Calculus of Probability. Holden-Day, San Francisco.

[9] Oodaira, H. and Yoshihara, K. (1971a). The law of the iterated logarithm for stationary processes satisfying mixing conditions. Ködai Math. Sem. Rep. 23 311-334.

[10] Oodaira, H. and Yoshinara, K. (1971b). Note on the law of the iterated logarithm for stationary processes satisfying mixing conditions. Ködai Math. Sem. Rep. 23 335-342.

[11] Philipp, W. (1969). The law of the iterated logarithm for mixing stochastic processes. Ann. Math. Statist. 40 1985-1991.

[12] Pratt, J. W. (1960). On interchanging limits and integrals. Ann. Math. Statist. 31 74-77.

[13] ReZnik, M. Kh. (1968). The law of the iterated logarithm for some classes of stationary processes. Theor. Probability Appl. 13 606-621.

[14] Scotr, D. J. (1971). Central limit theorems for martingales and for processes with stationary increments, using a Skorokhod representation approach. To appear in Adv. Appl. Probability.

[15] Stout, W. F. (1970). A martingale analogue of Kolmogorov's law of the iterated logarithm. Z. Wahrscheinlichkeitstheorie und Verw. Gebiete 15 279-290.

[16] Stout, W. F. (1970). The Hartman-Wintner law of the iterated logarithm for martingales. Ann. Math. Statist. 41 2158-2160.

[17] Strassen, V. (1964). An invariance principle for the law of the iterated logarithm. $Z$. Wahrscheinlichkeitstheorie und Verw. Gebiete 3 211-226.

[18] Strassen, V. (1967). Almost sure behavior of sums of independent random variables and martingales. Proc. Fifth Berkeley Symp. Math. Statist. Prob. 2 315-343. Univ. of California Press.

Department of Statistics

Australian National University

Box 4, P.O. Canberra, A.C.T. 2600

Australia 